\title{
Humean supervenience and tripartite entanglement relations
}

\section{Lorenzo Lorenzetti ${ }^{1}$}

Received: 25 March 2020/ Accepted: 12 October 2020/Published online: 4 November 2020

(C) The Author(s) 2020

\begin{abstract}
It has been argued that Humean Supervenience (HS) is threatened by the existence of quantum entanglement relations. The most conservative strategy for defending HS is to add the problematic entanglement relations to the supervenience basis, alongside spatiotemporal relations. In this paper, I'm going to argue against this strategy by showing how certain particular cases of tripartite entanglement states - i.e. GHZ states - posit some crucial problems for this amended version of HS. Moreover, I will show that the principle of free recombination - which is strictly linked to HS - is severely undermined if we add entanglement relations to the supervenience basis. I conclude that the conservative move is very unappealing, and therefore the defender of HS should pursue other, more controversial, strategies (e.g. committing to the nomological interpretation of the wave function).
\end{abstract}

Keywords Humean supervenience - Quantum mechanics · Entanglement relations $\cdot \mathrm{GHZ}$ state $\cdot$ Recombination $\cdot$ Locality

\section{Introduction}

This paper concerns the difficult relationship between Humean Supervenience and quantum entanglement. According to the thesis of Humean Supervenience (HS), the world is "a vast mosaic of local matters of particular fact" (Lewis 1986b: ix). That is, all there is in the world are distinct point-sized objects, which instantiate perfectly natural intrinsic properties and which are related by spatiotemporal relations.

Several authors have argued that this thesis is at least contingently false, in the light of modern physics. Indeed, it is often claimed that HS is directly in contrast with quantum mechanics. In particular, several philosophers have argued that the 
phenomenon of quantum entanglement entails that there are some wholes which have properties that are not reducible to the properties of their atomic parts, and that this proves that HS is wrong (see Teller (1986), Maudlin (2007), Karakostas (2009), Darby (2012), Calosi and Morganti (2016)).

There are a few ways in which HS can be defended. The less radical move we can pursue (although more radical strategies are viable) consists in adding the problematic entanglement relations to the supervenience basis-the class of entities upon which everything supervenes on-alongside fundamental intrinsic properties and spatiotemporal. By pursuing this strategy, we can account for the properties of entangled systems while relying only on relations between independent point-sized objects. Moreover, these relations would be somehow analogous to spatiotemporal relations. In this way, the Humean can argue that she has saved HS. Both Darby (2012) and Calosi and Morganti (2016) propose this kind of solution for the Humean. However, they are also critical with respect to this strategy. My aim for this paper will be to present two arguments against the aforementioned Humean strategy.

Firstly, I will analyse carefully HS, and I will state one necessary condition that has to be posited to save $\mathrm{HS}^{1}$ from being blatantly trivial. This is the condition according to which we can place only atom-to-atom relations in the supervenience basis, i.e. relations that do not have composite objects as their relata. Then, I will argue that entanglement relations fail to be atom-to-atom, at least in some particular cases of tripartite entanglement states, i.e. GHZ states. Moreover, I will point out that tripartite entanglement states violates also the locality constraint, which is a central part of the doctrine of HS.

Secondly, I will try to shed light on the problematic connection between entanglement relations and the Humean/Lewisian principle of free recombination, which is strictly related to HS. I will try to show that the strategy of adding entanglement relations to the supervenience basis clash with Lewis's idea that 'anything can coexist with everything' and that there are no necessary connections between the intrinsic nature of distinct entities. Such contrast highlights the fact that entanglement relations cannot be included within the supervenience basis, if we want to retain the principle of free recombination.

These arguments undermine severely the 'conservative' Humean strategy that I have sketched above. Still, the defender of HS can appeal to other-more radicalstrategies to save it. One option is to modify drastically HS to resist all the objections. However, as I will suggest in the paper, this would result in a complete betrayal of the original spirit of HS.

Indeed, the real alternatives for the Humean are the followings - which I shall introduce very briefly. Loewer (1996: 103-104) has claimed that HS can be saved if we maintain that the fundamental physical space is $3 \mathrm{~N}$-dimensional instead of 3-dimensional ${ }^{2}$ and we also drop orthodox quantum mechanics in favour of Bohmian mechanics. Similarly, Miller (2014) has argued that we can save HS by endorsing a combination of Bohmian mechanics and of the view according to which

\footnotetext{
1 Or, more generally, any kind of supervenience thesis.

2 See Albert (1996) for a classical defence of this view.
} 
the fundamental wave function is a law of nature, i.e. the nomological interpretation of the wave function. Also Esfeld (2014) and Bhogal and Perry (2017) defend HS by adopting the nomological account of the wave function (although independently from Bohmian mechanics).

These are, roughly, the solutions which are available to the defender of HS. However, they rely on controversial additional assumptions, and are thus more radical than the conservative strategy I have mentioned before. In fact, these strategies all hinges on questionable assumptions, which are still a matter of discussion. To put it roughly, the Humean would have to either endorse Bohmian mechanics or the nomological account of the wave function-and one can point out that it would be quite radical to endorse Bohmian mechanics only to save HS. Nonetheless, they are of course still possible options.

I will not focus on these strategies here. What I claim in the present paper is simply that, if one wants to defend HS, the conservative strategy of adding the entanglement relations to the supervenience basis is very problematic, and therefore one of the more radical options should be adopted instead.

\section{The humean mosaic and its relations}

The thesis of Humean Supervenience (HS) can be summed up by the following quotation from David Lewis:

Humean Supervenience [...] is the doctrine that all there is to the world is a vast mosaic of local matters of particular fact, just one little thing and then another. [...] We have geometry: a system of external relations of spatiotemporal distance between points. Maybe points of spacetime itself, maybe point-sized bits of matter or aether or fields, maybe both. And at those points we have local qualities: perfectly natural intrinsic properties which need nothing bigger than a point at which to be instantiated. For short: we have an arrangement of qualities. And that is all. There is no difference without difference in the arrangement of qualities. All else supervenes on that. (Lewis 1986b: ix-x).

As Weatherson claims, HS can be seen as composed by two distinct thesis:

The first is the thesis that $[\ldots]$ all the truths about a world supervene on the distribution of perfectly natural properties and relations in that world. The second is the thesis that the perfectly natural properties and relations in this world are intrinsic properties of point-sized objects, and spatiotemporal relations. (Weatherson 2016: §5).

On the one hand, the first thesis can be summed up by the slogan "truth supervenes on being". It concerns broadly the nature of the minimal basis of entities which grounds all the truths about a world. Here, we can take 'perfectly natural' as synonymous to 'fundamental'. Lewis, in fact, takes 'perfectly natural' properties to 
be those properties whose distribution entail all the truths about a world. ${ }^{3}$ If two possible worlds contain exactly the same natural properties and relations, arranged in the same way, then those worlds will be identical - or, better, exactly similar. Thus, the first thesis composing HS amounts to the claim that there is a minimal set of (kinds of) entities which entails all the truths about a world.

On the other hand, the second thesis specifies which kinds of entities fall into the minimal supervenience basis for the actual world. There are three ingredients there: point-sized objects; those intrinsic properties instantiated by them which are also perfectly natural; and spatiotemporal relations between those point-sized objects. However, Lewis is not clear about the meaning of the term 'point-sized'. In fact-as Weatherson (2015: 113) points out-when Lewis discusses about HS, he sometimes talks about intrinsic properties of point-sized objects and sometimes refers to local properties instead. However, these are not the same thing. On the one hand, we can read the expression 'intrinsic properties of point-sized objects' as referring to intrinsic properties of mereologically simple objects-as thus read 'point-sized' as 'mereologically simple'. On the other hand, as Butterfield (2006) argues, we can use the term 'local properties' to refer to properties that supervene on intrinsic features of arbitrarily small regions. And so, we could define HS in terms of intrinsic properties of arbitrary small regions. Both interpretations seems viable, and I will not be committed to one or another in this paper. Thus, in the following, when I will talk about 'point-size' or 'atomic' objects, this terms can be taken as referring to both mereologically simple objects and arbitrarily small regions of space.

One interesting point is that, according to HS, there is only one kind of relation that is perfectly natural and therefore fundamental (and, thus, present in the supervenience basis): spatiotemporal relations. Also, only intrinsic and local kind of properties are admitted. Lewis defines 'intrinsic' properties-modally-as follows:

The intrinsic properties of something depend only on that thing; whereas the extrinsic properties of something may depend, wholly or partly, on something else. If something has an intrinsic property, then so does any perfect duplicate of that thing; whereas duplicates situated in different surroundings will differ in their extrinsic properties. (Lewis 1983: 197).

At this point, a natural question arises: what is the criterion we should use to choose which properties and relations can be admitted to the supervenience basis and which can not? Let us focus on relations. Keep in mind that I will use the terms 'relation' and 'extrinsic property' interchangeably, and in the following I will formally express relations in terms of extrinsic properties. However, this is just a syntactical matter, and of course no substantive argument relies on this.

Lewis claims that the final criterion for picking out which kind of relations are fundamental should come from physics. In fact, he chooses spatiotemporal relations due to their-alleged-fundamental role in physics. However-taking a step back-are there a priori criteria for choosing which relations we should take as fundamental? That is, are there criteria which have to be satisfied independently from a posteriori physical reasons?

\footnotetext{
3 See Lewis (1983) and Dorr (2019).
} 
Some philosophers have pointed out some minimal a priori criteria which every relation should meet to be considered as part of a minimal supervenience basis. ${ }^{4}$ Let us start by considering two candidates extrinsic properties that are usually ruled out from the properties of the supevenience basis. The first property is described by Sider (2003: 140), while the second is mentioned by Merricks (1998: 60):

1. "Being part of a whole with such and such properties"

2. "Composing a square red object"

Actually, while the first is meant to be an extrinsic property, the second is expressed by Merricks as a relation that can hold between atoms. However, as I mentioned, a relation can be easily and uncontroversially translated as a (monadic) extrinsic property. Formally, this can be done via lambda abstraction, and this is precisely what I will do now. However, once again, keep in mind that this is just a syntactical move-the role of lambda abstraction is just that of making the present analysis more precise.

Thus, let us first translate 1 and 2 as follows (where $F$ is any property whatsoever that is possessed by the whole and not by any of its parts):

1. " $x$ is such that it is part of a $z$ that is $F$ "

2. " $x$ is such that together with $y$ composes a $F$ whole" 5

We can then try to translate these statements in the following formalized form (where $P$ is the parthood relation and ' $x+y$ ' is shorthand for ' $x$ and $y$ compose' $z$ ):

1. $\lambda \mathrm{x}(\exists \mathrm{z}(\mathrm{Pxz} \wedge \mathrm{Fz}))$

2. $\lambda x(\exists y \exists z(x+y=z \wedge F z))$

Put in this way, 1 and 2 express two properties that can be instantiated by a certain object $x$, which are both extrinsic properties according to Lewis's definition above ("the extrinsic properties of something may depend, wholly or partly, on something else").

As I have pointed out, Lewis includes only 'perfectly natural' or 'fundamental' properties within the supervenience basis linked to HS. Thus, it is quite obvious that this kind of properties would be ruled out from the basis in any case. However-and this is the crucial point-here I am claiming that, according to Merricks and Sider,

\footnotetext{
${ }^{4}$ More precisely, these criteria refer to microphysical supervenience and not directly to Humean supervenience. Merricks (1998: 59) defines Microphysical Supervenience (MS) as follows: "Necessarily, if atoms A1, through An compose an object that exemplifies intrinsic qualitative properties Q1 through Qn then atoms like A1 through An (in all their respective intrinsic qualitative properties), related to one another by all the same restricted atom-to-atom relations as A1 through An, compose an object that exemplifies Q1 through Qn.”. I will take HS as a specific version of MS, one that makes also explicit the nature of the fundamental microphysical properties and of the relations there are between them. Thus, since HS is a version of MS, the rules which apply to MS also apply to HS.

5 This is actually a literal and radical translation of a proposition of the kind "Composing an object with the property $\mathrm{F}$ ": indeed, $\mathrm{x}$ is such that together with $\mathrm{y}$ composes a $\mathrm{F}$ whole means that $\mathrm{x}$ and $\mathrm{y}$ are the only parts of a whole which instantiate the property F. Anyway, even if this does not represent the case of a square red object, this is just a radical example of the kind of case we are interested in, and so it will be equally suitable for my purposes.
} 
we have a priori reasons for not considering these properties, even in principle. That is-independently from any consideration of naturalness-this kind of properties cannot be considered as part of the supervenience basis, because they violate an implicit criteria which any version of supervenience thesis have to satisfy-i.e. any theory which tries to posit a minimal set of truths or entities upon which every truth supervenes on.

In fact, the problem with them is that they would make HS a trivial thesis:

It is trivial to say that the existence of a square red object composed of atoms supervenes on atoms' standing in the composing a square red object relation. (Merricks 1998: 60).

Abstracting away from the particular case, the problem with these relations is that among their relata there are not only point-sized objects (or arbitrarily small regions), but also composite wholes. Take 1 for example: $\lambda \mathrm{x}(\exists \mathrm{z}(\mathrm{Pxz} \wedge \mathrm{Fz}))$. This is a parthood relation between a point-sized object-which we can also call an 'atomic' object-and a composite whole instantiating certain properties. On the contrary, as Merricks argues, when we talk about relations within the supervenience basis, we should restrict our commitment to restricted atom-to-atom relations-i.e. relations between point-sized objects.

Why only atom-to-atom relations? Because, if we add to the basis relations which are not atom-to-atom, we would end up adding to the supervenience basis information about non-point-like objects which are supposed to supervene on the properties of spacetime points-and this is a dangerous slippery slope. In fact, if we were to add a kind of relation which is not 'atomic', we could in the same way add tons of them, up to the point where we would have every relation whatsoever in the supervenience basis. And, in that case, it would become trivial to say that all the truths about a world supervene on the distribution of the properties of the points of the mosaic and the relations between them, because we would already have 'all the truths' contained within the minimal basis.

Therefore, we can conclude that 'being atom-to-atom relations' is an a priori necessary condition which relations have to satisfy to be considered as parts of the supervenience basis. In the following, we shall see how-in certain situationsentanglement relations violate this necessary condition.

\section{Entanglement relations in the humean mosaic?}

The plan for this section is to present the argument against HS that relies on quantum mechanics, and in particular on the existence of entanglement relations. We shall see that quantum mechanics, according to which "certain physical systems - so called 'entangled systems' - exhibit correlations among their parts which are not reducible to the properties of these parts" (Calosi and Morganti 2016: 1177), seriously threatens the truth of HS. Let us briefly consider the argument in more details.

In quantum mechanics, a quantum state can be represented as a vector in a vector space (for example a unit vector $|\alpha\rangle$ in a vector space). A composite quantum system 
can be represented by "sums of tensor-products of the vector used to represent states of their components" (Darby 2012: 776). The peculiarity of quantum states (like the singlet state $\left.\frac{1}{\sqrt{2}}(|0\rangle \otimes|1\rangle-|1\rangle \otimes|0\rangle)\right)$ is that "it is just not possible to assign a state vector to either party separately, on its own" (ivi).

However, at this point the defender of HS can still point out there is a way to assign individual states to the parts, via the density operator $\rho$ which is determined by the description of the composite system. Taking the density operator of a composite system (composed for example by two particles), we can 'trace out' the density operator of one of the two parties to get the density operator corresponding to the other (single) party. Let us consider the following example. ${ }^{6}$

Consider four one-particle quantum systems $\mathrm{S} 1, \mathrm{~S} 1 *, \mathrm{~S} 2, \mathrm{~S} 2 * . \mathrm{S} 1$ and $\mathrm{S} 1 *$ are indiscernible and both are (when considered separately and pre-compositionally) in state (1):

$$
\frac{1}{\sqrt{2}}|0\rangle+\frac{1}{\sqrt{2}}|1\rangle
$$

Also S2 and S2* (when considered separately and pre-compositionally) are indiscernible and both are in state (2):

$$
\frac{1}{\sqrt{2}}|0\rangle+\frac{1}{\sqrt{2}}|1\rangle
$$

However, these systems can be added to constitute different composite systems, that is composite systems with different properties. S1 and S2 can compose the state S12 described in (3):

$$
|\phi\rangle_{12}=\frac{1}{\sqrt{2}}\left(|0\rangle_{1} \otimes|0\rangle_{2}+|1\rangle_{1} \otimes|1\rangle_{2}\right)
$$

whereas $\mathrm{S} 1 *$ and $\mathrm{S} 2 *$ can compose the state $\mathrm{S} 12 *$ described in (4):

$$
|\psi\rangle_{12}=\frac{1}{\sqrt{2}}\left(|0\rangle_{1} \otimes|1\rangle_{2}+|1\rangle_{1} \otimes|0\rangle_{2}\right)
$$

As I said, we can express the single parties that compose these composite systems via the density operator. We take the density operators $\rho^{\varphi}$ and $\rho^{\psi}$ of $(3,4)$ and we trace out a single party one at a time. At the end we have the following 'mixed states':

$$
\begin{aligned}
& \rho_{1}^{\phi}=\frac{1}{2}(|0\rangle\langle 0|+| 1\rangle\langle 1|)=\rho_{1 *}^{\psi} \\
& \rho_{2}^{\phi}=\frac{1}{2}(|0\rangle\langle 0|+| 1\rangle\langle 1|)=\rho_{2 *}^{\psi}
\end{aligned}
$$

\footnotetext{
${ }^{6}$ See Calosi and Morganti (2016: 1178-1194).
} 
Therefore, it can be observed that distinct but exactly similar components can give rise to discernible wholes. This is in fact a failure of HS, and more generally a failure of supervenience.

As Calosi and Morganti (2016) and Darby (2012) suggest, the most conservative solution for the Humean to deal with this issue is to add fundamental quantum entangled relations to the supervenience basis, turning HS into HS*:

(HS*) The world is a mosaic of fundamental, intrinsic point-like qualities and (dyadic) space time relations, plus entanglement relations; i.e., all facts either are or supervene on facts concerning such point-like qualities, together with (dyadic) space time and, possibly, entanglement relations. (Calosi and Morganti 2016: 1177).

Very roughly, those entanglement relations can be expressed by the predicates "having correlated spin" and "having anti-correlated (opposite) spin". Thus, we can formalize them as follows via lambda abstraction (in terms of extrinsic properties):

(a) $\lambda x(\exists y C x y)$

(b) $\lambda x(\exists y O x y)$

(where C means 'having correlated spin' and $\mathrm{O}$ means 'having opposite spin'). Thus, the state in (3) is determined by the reductive basis formed by (5), (6) and (a). Similarly, the state in (4) is determined by (5), (6) and (b) taken together. In this way, (3) and (4) are not determined by the same properties of the points in the mosaic, but are determined by such properties plus the entanglement relations, that are different for each pair of points. In this way, we have no discernible wholes that are determined by exactly similar components-and, moreover, the strategy just adopted seems at least prima facie correct and viable, considering what we have said so far about Humean supervenience.

However, even though the two entanglement relations in our example are 'atomto-atom relations', it is controversial to just take these relations and add them to the supervenience basis. In fact, my first objection will concern the possibility of entanglement relations between a single particle and a composite system of more than one particle. If such scenario is physically possible then we would have a fundamental relation that is not an atom-to-atom relation, and - given what I have said so far - this should already seem problematic. Secondly, contrarily to the classical spatiotemporal relations, entanglement relations seem to contrast with a key principle in Lewis's metaphysics, i.e. free recombinability. Thus, I am going to consider how the criteria for recombination cope with the kind of relation under investigation.

\section{Humean supervenience and the GHZ state}

Starting with the first argument, I claim that it is possible to have a fundamental entanglement relation between a single atomic particle and a whole composed by more than one particle. I will formalize this relation via lambda abstraction and show that clearly this is not an atom-to-atom relation. The example involves a set of 
quantum states called GHZ states, which are composed by three qubits maximally entangled.

Take three qubits constituting a GHZ state, and call the three qubits A, B and C. Since this is a GHZ state, we can say that $A$ is entangled with the composite system $\mathrm{BC}, \mathrm{B}$ is entangled with $\mathrm{AC}$ and $\mathrm{C}$ is entangled with $\mathrm{AB}$. However, the qubits are not entangled pairwise between them: $\mathrm{A}$ is not entangled with $\mathrm{B}$ alone, and $\mathrm{A}$ is also not entangled with $\mathrm{C}$ alone.

Formally, in quantum mechanics the measure of the entanglement of a tripartite state is expressed via the notion of 'tangle' $(\tau)$. More precisely, $\tau=0$ corresponds to an unentangled state, while $\tau=1$ corresponds to a completely entangled state. So, if the tangle of a state is equal to 0 the state is separable; on the contrary, if the tangle of a state is equal to 1 , such state is non-separable - i.e. maximally entangled. Thus, we can describe the relations between the elements of a GHZ state as follows. Consider the measurement of the entanglement of a 3-qubit quantum state expressed via the following equation:

$$
\tau \mathrm{A}(\mathrm{BC})=\tau \mathrm{AB}+\tau \mathrm{AC}+\tau \mathrm{ABC}
$$

Then,

Consider the Greenberger-Horne-Zeilinger (GHZ) state $\frac{1}{\sqrt{2}}(|000\rangle+|111\rangle)$.

For this state the tangle of each qubit with the rest of the system is 1 , the threeway tangle is also 1, and all the pairwise tangles are zero (the qubits in each pair are classically correlated but not entangled). Thus the equation above in this case becomes $1=0+0+1$. (Coffman et al. 2000: 10, italics added).

This shows that $\mathrm{A}$ is entangled with $\mathrm{BC}$, and that the quantum system composed by $\mathrm{A}, \mathrm{B}$ and $\mathrm{C}$ forms an entangled state. However, $\mathrm{A}$ is not individually entangled with $\mathrm{B}$, nor with $\mathrm{C}$. This implies that the entanglement between $\mathrm{A}$ and $\mathrm{BC}$ is not reducible to the entanglement between $\mathrm{A}$ and $\mathrm{B}$ and between $\mathrm{A}$ and $\mathrm{C}$, because such relations do not exist. Thus, if we want to add entanglement relations to the supervenience basis, in the case of a GHZ state we have to postulate a relation between a particle A and a composite system BC, which is clearly not an atom-toatom relation. That is-assuming that relations can be expressed as extrinsic properties via lambda abstraction-to account for the entanglement relations in our example we would have to attribute an extrinsic property of the following form to each of the parties $(\mathrm{A}, \mathrm{B}, \mathrm{C})^{7}$ :

(c) $\lambda x(\exists y \exists z \exists w(y+z=w \wedge E x w)$

Where ' $E$ ' is a generic entanglement relation. This means that, if we take entanglement relations in the supervenience basis in order to be able to correctly derive all the truths about the world from that basis, then in certain cases we would

\footnotetext{
7 Under the assumption that $\mathrm{y}$ and $\mathrm{z}$ in (c) do compose something. Notice, as I have pointed out before, that I am employing this formulation only to compare more precisely entanglement relations to relations such as "being part of a whole with such and such properties". However, nothing hinges on the lambda abstraction formulation: (c) is simply meant to represent a particular example of entanglement relation.
} 
be committed to relations which are not atom-to-atom. Indeed, in (c) above there is an object $w$, which is not an atomic object (indeed, it is composed by $y$ and $z$ ), that instantiates an (entanglement) relation with the atomic object $x$. And this is clearly not an atom-to-atom relation.

In this case, the problem is that we banned from the supervenience basis all the relations that are not atom-to-atom for a specific (a priori) reason-i.e. to avoid the risk of rendering HS a trivial thesis. Therefore, reintroducing them only to deal with particular examples like the GHZ states in quantum mechanics would definitely appear to be an ad hoc move-also because it is hard to see how we could state a clear demarcation criterion between which kind of non-atomic relation we can admit and which we cannot admit.

Nonetheless, there is a plausible objection to this last argument that the defender of HS could give, which I want to respond to.

As I said, we excluded 'not-atom-to-atom relations' to avoid the risk of trivializing the thesis. Because of this, and considering the peculiarity of the case concerning GHZ states, it would be either a dangerous slippery slope or an ad hoc move to reintroduce only some specific instances of 'not-atom-to-atom' relations within the supervenience basis only to save HS*. However, someone who wants to defend the doctrine of Humean supervenience could argue that a clear and not ad hoc criterion is actually available. That is, the demarcation criterion is offered by science itself. In Lewis's intentions, HS was meant to be a scientific-informed metaphysical theory. In Lewis own words:

The point of defending Humean Supervenience is not to support reactionary physics, but rather to resist philosophical arguments that there are more things in heaven and earth than physics has dreamt of. Therefore if I defend the philosophical tenability of Humean Supervenience, that defence can doubtless be adapted to whatever better supervenience thesis may emerge from better physics. (Lewis 1994: 474).

Thus, if quantum mechanics present us particular cases of 'not-atom-to-atom relations', we should look case by case and add to the supervenience basis of HS only the specific relations that we need. It is not ad hoc because the theory was supposed to look at what physics suggests from the beginning, and it is also not a slippery slope because a demarcation criterion is available.

However, even if it is possible to individuate in a principled way which 'notatom-to-atom' relations should be taken in the supervenience basis, GHZ states remains problematic for the following reasons. Whichever way we put it, the entanglement relations between the parts of these states will still remain relations between a point-sized object and a whole composed by two detached objects. In the relation between, for example, $\mathrm{A}$ and $\mathrm{BC}, \mathrm{B}$ and $\mathrm{C}$ can be located at very distant locations. Therefore, even though HS can be amended in a principled way to embed in its supervenience basis both atom-to-atom entanglement relations and also certain specific not-atom-to-atom entanglement relations-i.e. the ones characterizing GHZ states-the final result would represent a complete betrayal of the initial basic principles of HS. In fact, the Humean mosaic would contain among its basic constituents also objects which are neither 'point-sized' in the strict mereological 
sense, nor 'point-sized' in the loose sense of occupying 'arbitrarily small regions' of space. On the contrary, once again, the Humean mosaic would contain composite wholes composed by objects that can be located really far away one from the other. This is a straightforward violation of the locality intuition which is central to the original version of HS. In fact, HS pictured reality as fundamentally constituted by a constellations of individual points-in Lewis's words, "a vast mosaic of local matters of particular fact". If we have to add new kinds of relations and even composite wholes to the supervenience basis, how much is left of Humean supervenience?

However, the troubles for the Humean do not end here. In fact, together with the rejection of the idea of a mosaic composed merely by point-sized entities, another crucial element of Lewis's metaphysics-which is strictly related to HS-is threatened.

\section{Entanglement relations and free recombination}

The supervenience basis embedded within the doctrine of HS contains point-sized objects, the intrinsic and fundamental qualities possessed by them, and spatiotemporal relations between those points. In the last sections I have presented the problem posed by quantum entanglement relations to the thesis of HS, and I have considered one potential conservative answer that the defender of HS could provide to save HS from that threat-i.e. adding the problematic relations to the supervenience basis. However, I have tried to show that this strategy implies some unwelcomed consequences.

In this penultimate section I will present another additional challenge for the Humean. In fact, HS plays a crucial role also within another core principle of Lewis's metaphysics, namely the principle of free recombination. However, I will show that if we place entanglement relations in the supervenience basis, we have to give up this principle-or, at least, revise it heavily.

Let's start by considering again the central thesis behind HS: the idea that the world is "a vast mosaic of local matters of particular fact". Within Lewis's metaphysics, the concept of mosaic is strictly related to what he calls the principle of recombination. This principle is meant to be a guide to the space of metaphysical possibility. ${ }^{8}$ In Lewis's words:

We need a new way to say [...] that there are possibilities enough, and no gaps in logical space. To which end, I suggest that we look to the Humean denial of necessary connections between distinct existences. To express the plenitude of possible worlds, I require a principle of recombination according to which patching together parts of different possible worlds yields another possible world. Roughly speaking, the principle is that anything can coexist with

\footnotetext{
${ }^{8}$ Keep in mind that Lewis believes in modal realism, and thus he thinks that 'possible worlds' really exist out there just like the actual world does. Therefore, we should take the principle of recombination as an epistemological or heuristic principle, which simply gives us information about the space of metaphysical possibility. Moreover, we can apply the recombination principle to any possible world. That is, we can start from the fundamental entities of a possible world and recombine them to form other possible worlds.
} 
anything else, at least provided they occupy distinct spatiotemporal positions. Likewise, anything can fail to coexist with anything else. (Lewis 1986a: 87-88).

I shall call this the 'principle of free recombination', in order to highlight the fact that according to Lewis anything can coexist with anything. Indeed, as Lewis stresses, this principle "prohibits a necessary connection between the intrinsic character of a thing and the intrinsic character of a distinct thing with which it coexists" (Lewis 1986a: 181). Roughly, it tells us that any possible way in which the world can be arranged corresponds to a possible world.

However, it has to be stressed that the idea underlying the principle of free recombination is that any spatiotemporal distribution of perfectly natural-i.e. fundamental-properties is possible. That is, the principle of free recombination says that any distribution of perfectly natural properties possessed by point-sized objects standing in perfectly natural relations is possible. The principle is applied only to those kinds of entities because-given HS-those are the entities upon which every truth about the world supervenes on.

In other words, if you take every possible spatiotemporal arrangement of the actual fundamental point-sized objects (provided that they do not overlap) and every possible distribution of the actual fundamental intrinsic properties among those objects, you will have the full space of all the metaphysically possible worlds which instantiate the same kinds of fundamental properties as the actual world.

Thus, the combination of HS and of the principle of free recombination gives free recombinability of the perfectly natural properties of the fundamental objects with respect to the perfectly natural relations. But, if we turn HS into HS* by adding entanglement relations to the supervenience basis-i.e. by considering them among the perfectly natural relations-then free recombinability is threatened. That is, the space of metaphysically possible worlds would become narrower, because not every recombination of perfectly natural properties would be possible anymore. ${ }^{9}$

To better illustrate this claim, let us consider an example. Take two particles, A and $\mathrm{B}$, which are related by the entanglement relation of 'having anti-correlated spin', and which possess definite and intrinsic position properties. Then, take two other particles $\mathrm{C}$ and $\mathrm{D}$, which are unentangled and which possess definite and intrinsic spin properties.

In this scenario, we cannot recombine the intrinsic properties of those particles freely. In fact, suppose that we switched the intrinsic profiles of $\mathrm{A}$ and $\mathrm{B}$ with the ones of $\mathrm{C}$ and $\mathrm{D}$. If we did, we would have a particle, $\mathrm{A}$, that has both a determined spin (say, 'spin up') and that is in an entanglement relation with B with respect to spin. Thus, if-after a measurement-we had measured 'spin up' on B, we would be compelled to say that A possesses a 'spin down' property, since this follows from the very nature of quantum entanglement. At that point, A would instantiate both a 'spin up' and a 'spin down' property. But, of course, this is a contradiction.

\footnotetext{
9 Also Karakostas (2009: 18) and Calosi and Morganti (2016: 1186), in a similar vein, stress the fact that entanglement relations do not sit well with Lewis's 'atomistic' picture consisting of unconnected and independent point-sized objects and with the principle of free recombination.
} 
This means that-due to the presence of fundamental entanglement relations-we cannot recombine the qualitive profiles of the fundamental constituents of the world freely. In the scenario of our example, in fact, we cannot switch the intrinsic nature of the two pairs of particles, since that would give rise to logical contradictions, in certain cases. This is due to the fact that when two particles instantiate an entanglement relation their intrinsic profiles are inter-dependent.

In other words, entanglement relations are fundamental external relations which constrain the intrinsic properties of their relata. That is, they establish necessary connections between the intrinsic characters of their (distinct) relata. This means that the principle according to which there are no necessary connections between the intrinsic character of a thing and the intrinsic character of a distinct thing fails, and therefore also free recombinability fails, if entanglement relations belong to the category of the perfectly natural relations.

In conclusion, if we want to keep the Lewisian thesis of free recombination, then there is no place for entanglement relations among the fundamental elements of the Humean mosaic. This means that the strategy of adding the problematic entanglement relations among the fundamental relations constrain severely the principle of free recombination.

Naturally, one solution for the Humean could be to modify the principle of recombination. She could maintain that, due to the existence of entanglement relations, there are some constraints that we have to impose on the possible recombinations of intrinsic properties. However, this would be of course a betrayal of the original spirit of the theory.

Consider what I have shown so far. If the Humean chooses to defend HS by adding entanglement relations to the supervenience basis - together with spatiotemporal relations - she would be compelled to maintain also that: (1) some composite 'non-local' wholes are part of the supervenience basis, contrarily to the initial assumption that the Humean mosaic is composed only by point-sized local objects, and (2) that we cannot freely recombine the intrinsic properties instantiated by the objects composing the supervenience basis. Naturally this amended version of HS is still a coherent theory. However, how much is left of the original thesis? Humean supervenience would be saved, but at the cost of not being really 'Humean' anymore.

\section{Conclusion}

In this paper I have explored the difficult relationship between the David Lewis's thesis of Humean supervenience and quantum entanglement relations. In particular, I have shown how entanglement relations threaten HS and how the Humean can deal in a straightforward and conservative way with that issue. Then, I have argued that that strategy entails several unwelcomed consequences. Very roughly, it results in a complete betrayal of HS original spirit. That is, if the Humean adds entanglement relations to the supervenience basis, she cannot maintain that the world is a vast mosaic of disconnected and local matters of particular fact anymore. 
Given this, I conclude that the conservative strategy for saving HS is very much unappealing, and therefore no Humean would plausibly endorse it. Therefore, the defender of HS seems to be forced to adopt one of the more complex and controversial solutions which I have briefly presented in the first section.

Acknowledgements I am deeply grateful to Claudio Calosi for all his useful comments. Thanks also to Matteo Morganti, Stefan Wolf and to one anonymous referee for their help.

Open Access This article is licensed under a Creative Commons Attribution 4.0 International License, which permits use, sharing, adaptation, distribution and reproduction in any medium or format, as long as you give appropriate credit to the original author(s) and the source, provide a link to the Creative Commons licence, and indicate if changes were made. The images or other third party material in this article are included in the article's Creative Commons licence, unless indicated otherwise in a credit line to the material. If material is not included in the article's Creative Commons licence and your intended use is not permitted by statutory regulation or exceeds the permitted use, you will need to obtain permission directly from the copyright holder. To view a copy of this licence, visit http:// creativecommons.org/licenses/by/4.0/.

Funding Open access funding provided by Università della Svizzera italiana.

\section{References}

Albert DZ (1996) Elementary quantum metaphysics. Bohmian mechanics and quantum theory: an appraisal. Springer, Dordrecht, pp 277-284

Bhogal H, Perry Z (2017) What the humean should say about entanglement. Noûs 51(1):74-94

Butterfield J (2006) Against pointillisme about mechanics. Br J Philos Sci 57(4):709-753

Calosi C, Morganti M (2016) Humean supervenience, composition as identity and quantum wholes. Erkenntnis 81(6):1173-1194

Coffman V, Kundu J, Wootters WK (2000) Distributed entanglement. Phys Rev A 61(5):052306

Darby G (2012) Relational holism and Humean supervenience. Br J Philos Sci 63(4):773-788

Dorr, C. (2019) Natural Properties. The Stanford Encyclopedia of Philosophy (Fall 2019 Edition), Edward N. Zalta (ed.), URL $=<$ https://plato.stanford.edu/archives/fall2019/entries/naturalproperties/>.

Esfeld M (2014) Quantum Humeanism, or: physicalism without properties. Philos Q 64(256):453-470

Karakostas V (2009) Humean supervenience in the light of contemporary science. Metaphysica 10(1):1-26

Lewis DK (1983) Extrinsic properties. Philos Stud Int J Philos Anal Tradit 44(2):197200

Lewis DK (1986a) On the plurality of worlds. Blackwell, UK

Lewis DK (1986b) Philosophical papers:, II. Oxford University Press, USA

Lewis PJ (2016) Quantum ontology: a guide to the metaphysics of quantum mechanics. Oxford University Press, USA

Loewer B (1996) Humean supervenience. Philos Top 24(1):101-127

Maudlin T (2007) The metaphysics within physics. Oxford University Press, USA

Merricks T (1998) Against the doctrine of microphysical supervenience. Mind 107(425):5971

Miller E (2014) Quantum entanglement, bohmian mechanics, and humean supervenience. Australas J Philos 92(3):567-583

Sider T (2003) Merricks on microphysical supervenience. Philos Phenomenol Res 66:139-149

Teller P (1986) Relational holism and quantum mechanics. Br J Philos Sci 37(1):71-81

Weatherson B (2015) Humean supervenience. In: Loewer B, Schaffer J (eds) A companion to David Lewis. Wiley, Oxford UK, pp 101-115 
Weatherson, B. (2016) David Lewis. The Stanford Encyclopedia of Philosophy (Winter 2016 Edition), Edward N. Zalta (ed.), URL $=<$ https://plato.stanford.edu/archives/ win2016/entries/david-lewis/>.

Publisher's Note Springer Nature remains neutral with regard to jurisdictional claims in published maps and institutional affiliations.

\section{Affiliations}

\section{Lorenzo Lorenzetti ${ }^{1}$}

$\bowtie$ Lorenzo Lorenzetti

lorenzo.lorenzetti@usi.ch

1 Università della Svizzera italiana, Lugano, Switzerland 